\title{
Modified Subtraction Coronary CT Angiography with a Two-Breathhold Technique: Image Quality and Diagnostic Accuracy in Patients with Coronary Calcifications
}

\author{
Weifeng Guo, $M D^{1,2}$, Pratik Tripathi, $M D^{1}$, Shan Yang, $M D^{1}$, Juying Qian, MD, PhD ${ }^{3}$, Bimal Rai, $M D^{4}$, \\ Mengsu Zeng, MD, PhD ${ }^{1,2}$ \\ Departments of ${ }^{1}$ Radiology and ${ }^{3}$ Cardiology, Zhongshan Hospital, Fudan University, Shanghai, China; ${ }^{2}$ Shanghai Institute of Medical Imaging, \\ Shanghai, China; 'Department of Radiology, Tongji Hospital, Tongji Medical College, Huazhong University of Science and Technology, Wuhan, China
}

Objective: To evaluate a modified subtraction coronary computed tomography angiography (CCTA) technique with a twobreathhold approach in terms of image quality and stenosis grading of calcified coronary segments and in the detection of significant coronary stenosis in segments with severe calcification.

Materials and Methods: The Institutional Review Board approved this study, and all subjects provided written consent. A total of 128 patients were recruited into this trial, of which 32 underwent subtraction CCTA scans and invasive coronary angiography (ICA). The average Agatston score was $356 \pm 145$. In severely calcified coronary segments, the presence of significant $(>50 \%)$ stenosis was assessed on both conventional CCTA and subtraction CCTA images, and the results were finally compared with ICA findings as the gold standard.

Results: For severely calcified segments, the image quality in conventional CCTA significantly improved from $2.51 \pm 0.98$ to $3.12 \pm 0.94$ in subtraction CCTA $(p<0.001)$. In target segments, specificity $(70 \%$ vs. $87 \% ; p=0.005)$ and positive predictive value $(61 \%$ vs. $79 \% ; p<0.01)$ were improved using subtraction CCTA in comparison with conventional CCTA, with no loss in the negative predictive value. The segment-based diagnostic accuracy for detecting significant stenosis was significantly better in subtraction CCTA than in conventional CCTA (area under the receiver operating characteristic curve, 0.94 vs. 0.85; $p=0.03)$.

Conclusion: This modified subtraction CCTA method showed lower misregistration and better image quality in patients with limited breathhold capability. In comparison with conventional CCTA, modified subtraction CCTA would allow stenosis regrading and improve the diagnostic accuracy in coronary segments with severe calcification.

Keywords: 320-detector row CT; Coronary CT angiography; Plaque calcification; Subtraction

\section{INTRODUCTION}

Multidetector coronary computed tomography angiography (CCTA) is the first-choice examination modality for patients suspected of having coronary artery disease (1-3). However, blooming and beam-hardening artifacts due to the presence of severe coronary calcification or implanted stents are still major problems for accurate evaluation of segment- based coronary stenosis $(4,5)$. Therefore, some studies have reported that CCTA should not be performed as the next step in the diagnostic workup of patients with a high coronary artery calcification score $(6,7)$.

Recently, a new postprocessing technique called subtraction CCTA has been introduced. In subtraction CCTA, plaque calcifications in the coronary arteries were eliminated by subtracting precontrast CT image data from

Received November 30, 2018; accepted after revision March 20, 2019.

This study has been performed with technological support from Canon Medical Systems, China.

Corresponding author: Mengsu Zeng, MD, PhD, Department of Radiology, Zhongshan Hospital, Fudan University, No. 180, Fenglin Avenue, Xuhui District, Shanghai 200032, China.

- Tel: (86021) 64041990 • Fax: (86021) 64041990 • E-mail: zengmengsu@outlook.com

This is an 0pen Access article distributed under the terms of the Creative Commons Attribution Non-Commercial License (https:// creativecommons.org/licenses/by-nc/4.0) which permits unrestricted non-commercial use, distribution, and reproduction in any medium, provided the original work is properly cited. 
postcontrast CCTA image data. Promising results have been reported by applying subtraction CCTA in patients with severe coronary calcifications or stents (8-15).

In subtraction CCTA, one-breathhold and two-breathhold scanning techniques have been used. In previous studies, researchers mainly focused on a long-lasting one-breathhold scan protocol in subtraction CCTA (9-13). The onebreathhold approach requires a relatively longer breathhold for the acquisition of precontrast and postcontrast image data. Many elderly patients with high coronary calcification suffer from obstructive lung disease and may not be able to hold their breath for such a long time, and thus, a twobreathhold approach would be suitable in this scenario. Nevertheless, misregistration artifacts have been shown to be pronounced due to misalignment between contrast and mask images during the subtraction process, which has been observed in approximately $50 \%$ of all target segments using the original two-breathhold scanning protocol $(14,15)$.

To further minimize misregistration artifacts, we propose a modified two-breathhold subtraction approach (Fig. 1) in patients who are unable to perform a long breathhold. The aim of our study was to evaluate the applicability of subtraction CCTA using this modified scanning protocol in terms of image quality and stenosis grading of calcified coronary segments and in the detection of significant coronary stenosis in coronary segments with severe calcification in comparison with conventional CCTA.

\section{MATERIALS AND METHODS}

\section{Study Population}

All patients who met the inclusion criteria agreed to

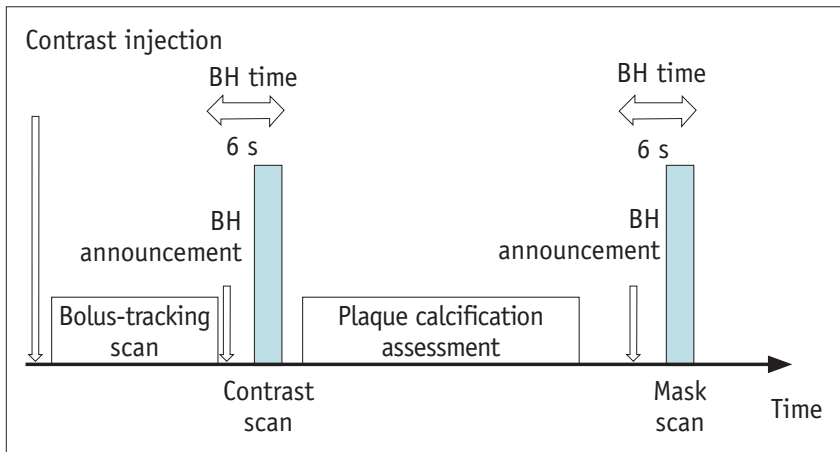

Fig. 1. Scan protocol for imaging acquisition for modified subtraction CCTA.

$\mathrm{BH}$ time is interval between end of $\mathrm{BH}$ announcement to end of contrast scan, and also to end of mask scan respectively. $\mathrm{BH}=$ breathholding, CCTA = coronary computed tomography angiography participate in the study and signed an informed consent form. The study protocol was approved by the Institutional Review Board from our hospital.

Patients with suspected or known coronary artery disease were prospectively enrolled from August 2016 to December 2017. For cases involving routine CCTA, exclusion criteria were a history of allergic reaction to iodinated contrast agents, arrhythmia, previous cardiac surgery, evidence of acute coronary syndrome, New York Heart Association functional class III or IV heart failure, aortic stenosis, and intolerance to beta-blockers.

For subtraction CCTA scans, the inclusion criteria were heart rate $(H R) \leq 65$ beats per minute $(\mathrm{bpm})$ after oral administration of beta-blocker (Betaloc, $25 \mathrm{mg}$; AstraZeneca, London, UK) or basal $\mathrm{HR} \leq 65 \mathrm{bpm}$ and coronary artery calcification detected in the proximal segments. Proximal coronary segments were defined as previously described (9). Patients were excluded for further mask scans due to motion artifacts in contrast images.

During the study period, a total of 164 patients underwent subtraction CCTA due to coronary artery calcification or stent placement. Of these 164 patients, 36 patients (33 with coronary stents, 2 with motion artifacts, and 1 with insufficient coronary lumen contrast enhancement) were excluded from the study. Finally, 128 patients who had undergone subtraction CCTA scanning were included for further analysis, of which 32 (25\%) underwent CCTA with invasive coronary angiography (ICA) as the reference standard to determine the diagnostic value.

ICA

ICA using the standard technique was performed in clinically indicated subjects within 60 days of subtraction CCTA scanning. ICA (Anigostar Plus; Siemens Healthineers, Berlin, Germany) was performed using standardized angiographic techniques. Quantitative coronary analysis (QCA) was performed using a software program (Syngo fastView, VX57I33; Siemens Healthineers) by a cardiologist with more than 15 years of experience. Significant stenosis was defined as $>50 \%$ luminal reduction in the most severe stenosis in the calcified segments.

\section{CCTA Data Acquisition}

All scans were performed using a 320-detector row CT scanner (Aquilion ONE VISION Edition; Canon Medical Systems, Otawara, Japan) with 0.5-mm detector elements and a gantry rotation time of 275 ms. Images 
were reconstructed with a $512 \times 512$ matrix and 0.5$\mathrm{mm}$ thickness by using standard kernel FCO9 iterative reconstruction (AIDR 3D; Canon Medical Systems). Betablockers were given when the resting HR was above $65 \mathrm{bpm}$ unless patients had contraindications. Sublingual shortacting nitroglycerin (0.5 mg; Yimin Pharma, Beijing, China) was routinely given during data acquisition.

Noncontrast CT scanning was performed for all patients to determine the calcification score using the method described by Agatston et al. (16). Noncontrast cardiac CT scans were obtained with a tube voltage of $120 \mathrm{kV}$ and a tube current of $50 \mathrm{~mA}$. Mid-diastolic or end-systolic images were acquired using the prospective gating method. The scan range included the region from the origin of the aorta to the apex of the heart. The reconstruction slice thickness was $3 \mathrm{~mm}$.

We adopted a modified two-breathhold approach in this study (Fig. 1). After the contrast imaging acquisition, the investigator determined whether the target coronary segments needed the mask images for postprocessing subtraction. It took at least 60 seconds after the contrast injection until the next noncontrast scan. Theoretically, there was no contrast residue in the coronary artery system and left cardiac chamber during mask scanning. Both the contrast and mask image data were acquired by using a prospective electrocardiography triggering technique between $65 \%$ and $80 \%$ of the RR interval. Scanning was performed with a tube voltage of $120 \mathrm{kVp}$ and a rotation time of $275 \mathrm{~ms}$. The tube current setting was selected based on the value calculated by the automatic exposure control function (17) with the standard deviation (SD) value set to $20 \mathrm{HU}$.

The contrast-enhanced scan was performed using iodinated contrast medium with an iodine concentration of 370 mg/mL (Ultravist ${ }^{\circledR}$ iopromide-370; Bayer AG, Berlin, Germany) injected at a rate of body weight $(\mathrm{kg}) \times 0.06 \mathrm{~mL} /$ $\mathrm{s}$ in $10 \mathrm{~s}$ (fixed), followed by a $30-\mathrm{mL}$ saline chaser bolus. The effective radiation dose was estimated based on the dose-length product (DLP, mGy $x \mathrm{~cm}$ ) by using the formula of effective radiation dose $=\mathrm{DLP} \times \kappa$, where $\kappa=0.014 \mathrm{mSV}$ $x \mathrm{mGy}^{-1} \times \mathrm{cm}^{-1}(18)$.

\section{Subtraction Postprocessing}

Subtraction was performed using a dedicated algorithm "volumetric CT digital subtraction angiography" (19) that was available on the scanner console. First, a global nonrigid registration was performed, followed by a local rigid registration for the targeted coronary segment. Thus, the results obtained reflected only the contrast filling the coronary artery without calcification.

\section{Image Analysis}

The acquired conventional and subtraction CCTA images were transferred to a workstation (Vitrea, version 6.5.3; Vital, Minneapolis, MN, USA) to generate a curved planar reformation image and a cross-sectional image. The coronary arteries were divided into 17 segments according to the American Heart Association classification. Plaque calcification within each coronary segment was determined as follows: in addition to the most severe plaque calcification identified by CCTA using a cross-sectional grading method as previously described (20), we assessed the plaque calcification associated with the maximum crosssectional arc within each segment as defined by CCTA for this investigation. To analyze the stenosis in the targeted segment, the minimum lumen diameter was measured at the site with the highest degree of arc calcifications within each segment. On the basis of the extent of segment calcification determined using an adapted cross-sectional arc calcification method (21), the targeted calcified plaques were classified as mild calcification (cross-sectional arc calcium $<90^{\circ}$ ), moderate calcification (cross-sectional calcium arc $90^{\circ}$ $180^{\circ}$ ), and severe calcification (cross-sectional calcium arc $>180^{\circ}$ ) (Fig. 2).

Segmental image quality was evaluated for each calcified segment using a 4-point scale: 1 = uninterpretable, 2 = poor image quality, 3 = adequate image quality, $4=$ good image quality. Segments with two points due to distinct misregistration were deemed nondiagnostic and were considered to be equivalent to severe stenosis for subsequent analysis.

Quantitative assessment of the stenosis was performed in cross-sectional and longitudinal projections by two experienced cardiac radiologists with more than 10 years of experience. The radiologists were blinded to the results of ICA but were aware of the clinical history of the patients. Stenosis was graded as minimal, mild, moderate, and severe, corresponding respectively to stenosis of $\leq 25 \%$, $26-50 \%, 51-75 \%$, and $\geq 76 \%$, as recommended by the Society of Cardiovascular Computed Tomography (22). Discrepancies were settled by consensus. Note that if the targeted segment was judged to be non-assessable, the image findings were classified as severe stenosis. 


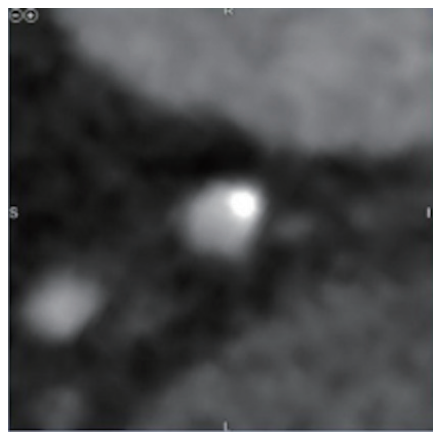

A
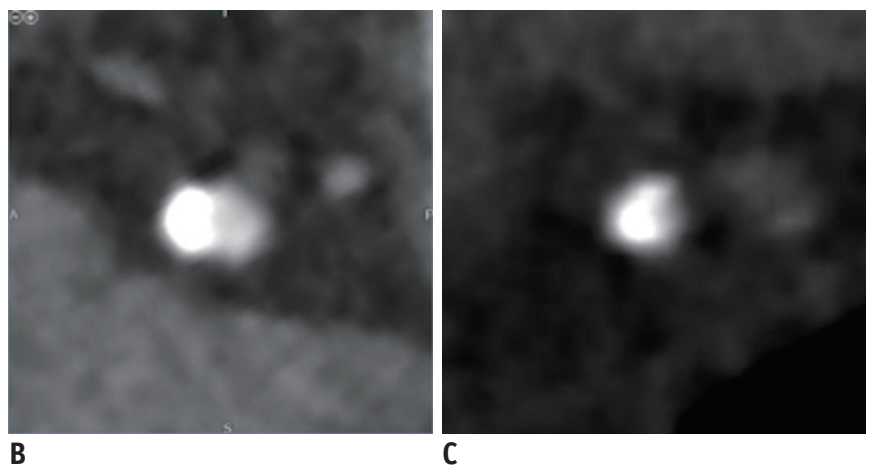

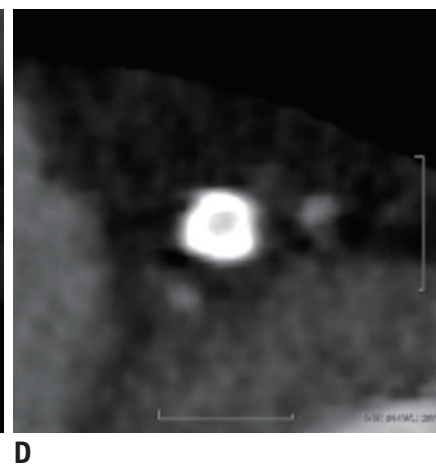

Fig. 2. Examples of coronary atherosclerotic plaque calcification by CT images.

A. Mildly calcified (cross-sectional arc calcium $<90^{\circ}$ ). B. Moderately calcified (cross-sectional arc calcium $90^{\circ}-180^{\circ}$ ). C, D. Severely calcified (cross-sectional arc calcium $>180^{\circ}$ ) segments. Segment calcification was measured by using cross-sectional arc method.

\section{Statistical Analysis}

We evaluated the data on a segment level. The average image quality scores between conventional and subtraction CCTA images were compared using a paired $t$ test. The diagnostic vs. nondiagnostic image quality frequencies were compared using the McNemar test. The kappa coefficient was used to measure agreement between conventional and subtraction CCTA for targeted segment stenosis grading. For all the ICA examinations performed, the conventional and subtraction CCTA diagnostic accuracies (sensitivity, specificity, positive predictive value, and negative predictive value) for detecting significant stenosis (> $50 \%$ ) were assessed in segments with severe calcification, and $p$ values for comparison between diagnostic tests were calculated by the McNemar test. To assess diagnostic accuracy, the area under the curve (AUC) of the receiver operating characteristic (ROC) was calculated for both conventional CCTA and subtraction CCTA. The method proposed by DeLong et al. (23) was used for comparison of the two ROC curves. Statistical analyses were performed using the Statistical software MedCalc version 11.5.0.0 (MedCalc Software, 0stend, Belgium). Metric variables were expressed as the mean $\pm \mathrm{SD}$. All tests were two-tailed with a significance threshold of $p$ values less than 0.05 and a confidence interval (CI) of 95\%.

\section{RESULTS}

The clinical, demographic, and radiological characteristics of the 128 included patients are shown in Table 1. Of these patients, 25\% (32/128) with 58 severely calcified segments further received ICA as the reference standard for detection of significant stenosis to evaluate the diagnostic value of this modified subtraction technique. The average Agatston
Table 1. Patient Characteristics $(n=128)$

\begin{tabular}{|c|c|}
\hline Characteristics & Values \\
\hline \multicolumn{2}{|l|}{ Age (years) } \\
\hline Mean \pm SD & $67 \pm 8$ \\
\hline Range & $48-84$ \\
\hline \multicolumn{2}{|l|}{$\operatorname{Sex}(n)$} \\
\hline Male & $80(62.5 \%)$ \\
\hline Female & $48(37.5 \%)$ \\
\hline \multicolumn{2}{|l|}{ Body mass index $\left(\mathrm{kg} / \mathrm{m}^{2}\right)$} \\
\hline Mean \pm SD & $23.6 \pm 3.8$ \\
\hline Range & $17.2-30.0$ \\
\hline \multicolumn{2}{|l|}{ Coronary risk factors $(\mathrm{n})$} \\
\hline Hypertension & $70(54.7 \%)$ \\
\hline Diabetes & $42(32.8 \%)$ \\
\hline Hypercholesterolemia & $62(48.4 \%)$ \\
\hline Smoking & $48(37.5 \%)$ \\
\hline Previous myocardial infarction & $8(6.3 \%)$ \\
\hline \multicolumn{2}{|l|}{ Coronary calcium score } \\
\hline Mean \pm SD & $356 \pm 145$ \\
\hline Range & $36-849$ \\
\hline Median & 348 \\
\hline Interquartile range & $132-568$ \\
\hline \multicolumn{2}{|l|}{ HR at contrast scan (bpm) } \\
\hline Mean \pm SD & $57 \pm 6$ \\
\hline Range & $46-63$ \\
\hline \multicolumn{2}{|l|}{ HR at mask scan (bpm) } \\
\hline Mean \pm SD & $57 \pm 8$ \\
\hline Range & $47-65$ \\
\hline \multicolumn{2}{|c|}{ Estimated effective radiation dose (mSv) } \\
\hline Mean \pm SD & $5.5 \pm 1.7$ \\
\hline Range & $3.5-9.2$ \\
\hline
\end{tabular}

$\mathrm{bpm}=$ beats per minute, $\mathrm{HR}=$ heart rate, $\mathrm{SD}=$ standard deviation

score was $356 \pm 145$. The total effective radiation dose for the subtraction CCTA scan was $5.3 \pm 1.2 \mathrm{mSv}$. The mean HR was $57 \pm 6 \mathrm{bpm}$ (range, 46-63 bpm) during the contrast scan and $57 \pm 8 \mathrm{bpm}$ (range, 47-65 bpm) during the mask scan. 
Image Quality Analysis between Conventional CCTA and Subtraction CCTA

A total of 128 patients with 564 calcified coronary segments underwent subtraction scanning. With regard to the global calcified segments, the mean coronary image quality score with conventional CCTA was $3.30 \pm 0.86$ and $3.56 \pm 0.62$ with subtraction CCTA $(p<0.01)$. On the basis of conventional CCTA images, $106(82.8 \%)$ patients with $216(38.3 \%)$ segments were judged to have mild calcification, and 94 (73.4\%) patients with 185 (32.8\%) segments were judged to have moderate calcification. However, image quality in subtraction CCTA did not change significantly in segments with mild calcification (3.68 \pm 0.54 to $3.71 \pm 0.49, p=0.68)$ and segments with moderate calcification ( $3.49 \pm 0.65$ to $3.56 \pm 0.59, p=0.48)$ (Fig. 3 ). Eighty-four of the 128 subjects were judged to have severe calcification, with severe calcification observed in $163(28.9 \%)$ of the total segments. In the segments showing severe calcifications, after the subtraction of beam-hardening artifacts, the image quality significantly improved from $2.51 \pm 0.98$ to $3.12 \pm 0.94(p<0.001)$ (Fig. 3). Misregistration artifacts were recorded in 17 segments $(8 \%)$ in the group with mild calcification, 24 segments $(13 \%)$ in the group with moderate calcification, and 42 segments $(26 \%)$ in the group with severe calcification.

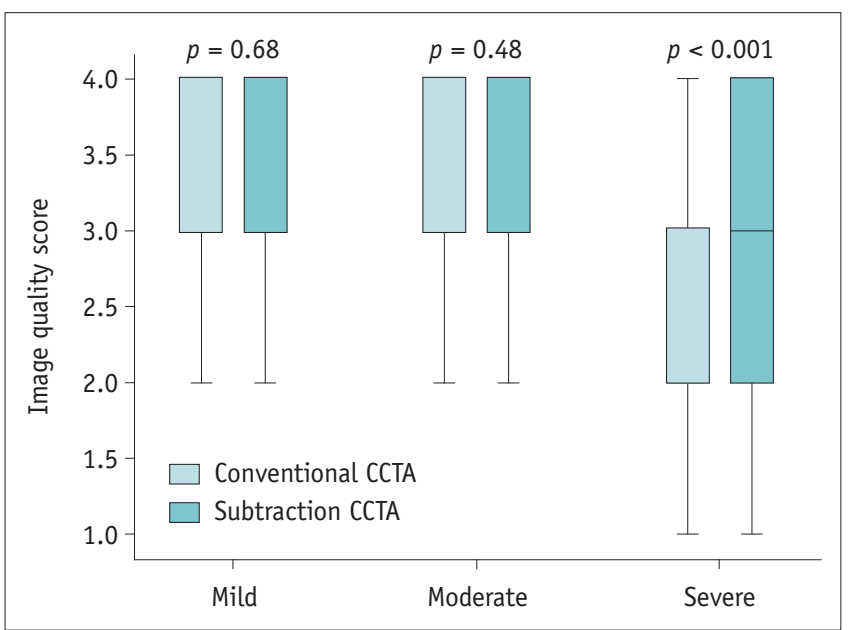

Fig. 3. Image quality of conventional and subtraction computed tomography angiography in different degrees of segment-based plaque calcifications. Image quality in subtraction CCTA is significantly improved in segments with severe calcification $(2.51 \pm 0.98$ to $3.12 \pm 0.94, p<0.001)$. However, image quality is only slightly improved in segments with mild calcification (3.68 \pm 0.54 to $3.71 \pm 0.49)$ and segments with moderate calcification (3.49 \pm 0.65 to $3.56 \pm 0.59)$, but not significantly $(p=0.68$ and $p=0.48$, respectively). Image quality score: $1=$ uninterpretable, $2=$ poor image quality, 3 = adequate image quality, 4 = good image quality.
Stenosis Regrading with Subtraction CCTA in Comparison with Conventional CCTA

For the stenosis grading in conventional CCTA and subtraction CCTA, the kappa index was 0.88 (95\% CI, $0.80-0.95 ; p<0.001)$ in the group with mild calcification and 0.84 (95\% CI, 0.75-0.92; $p<0.001)$ in the group with moderate calcification. We observed good agreement values between the mild and moderate calcification groups. Nevertheless, for segments with severe calcification, there was a fair correlation between conventional CCTA and subtraction CCTA for the assessment of stenotic grading, with a kappa index of 0.57 (95\% CI, 0.44-0.70; $p<0.001$ ). Of the 163 segments with severe calcification, 52 (32.0\%) were changed to a different stenotic class after subtraction, among which 21 (40.4\%) were downgraded from moderate to mild stenosis, $17(32.7 \%)$ were downgraded from severe to moderate stenosis, $8(15.4 \%)$ were downgraded from severe to mild stenosis, and $6(11.5 \%)$ were upgraded from mild to moderate stenosis. Segments showing grade changes in the mild and moderate calcification groups were less frequent at $11.1 \%(24 / 216)$ and $16.2 \%$ (30/185), respectively. Figure 4 shows the cases of stenosis regrading after subtraction.

\section{Subanalyses of Diagnostic Accuracy in Target Segments} with Severe Calcifications

A total of $25 \%(32 / 128)$ of patients with 58 severely calcified lesions underwent subtraction CCTA scans followed by ICA. In the group with severe calcification, nondiagnostic segments with conventional CCTA were obtained in 33\% (19/58) of the cases, and after subtraction, the corresponding percentage was 10\% (6/58) $(p<0.01)$. Statistical performance of conventional CCTA and subtraction CCTA to detect coronary artery stenosis $>50 \%$ is shown in Table 2. The AUC for the diagnosis of significant stenosis as determined by QCA (Fig. 5) was 0.94 (95\% CI, $0.86-1.00)$ in subtraction CCTA, which was higher ( $95 \%$ CI, 0.74-0.96) than that for conventional CCTA $(0.85 ; p=0.03)$. An example is shown in Figure 6.

\section{DISCUSSION}

The current study provided a preliminary assessment of the practicability of modified subtraction CCTA with a two-breathhold method in patients unable to maintain a sufficiently long-lasting single breathhold. With this modified approach, image quality was significantly better in 


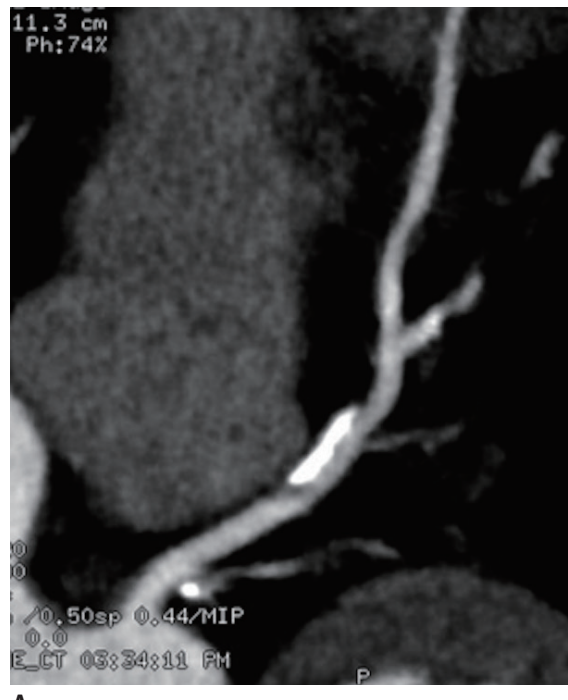

A

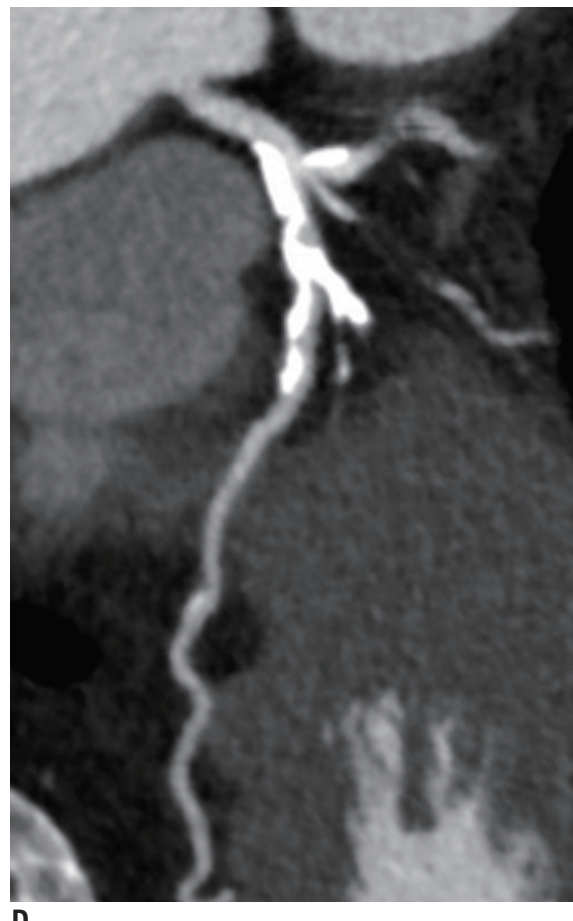

D

$\mathrm{E}$
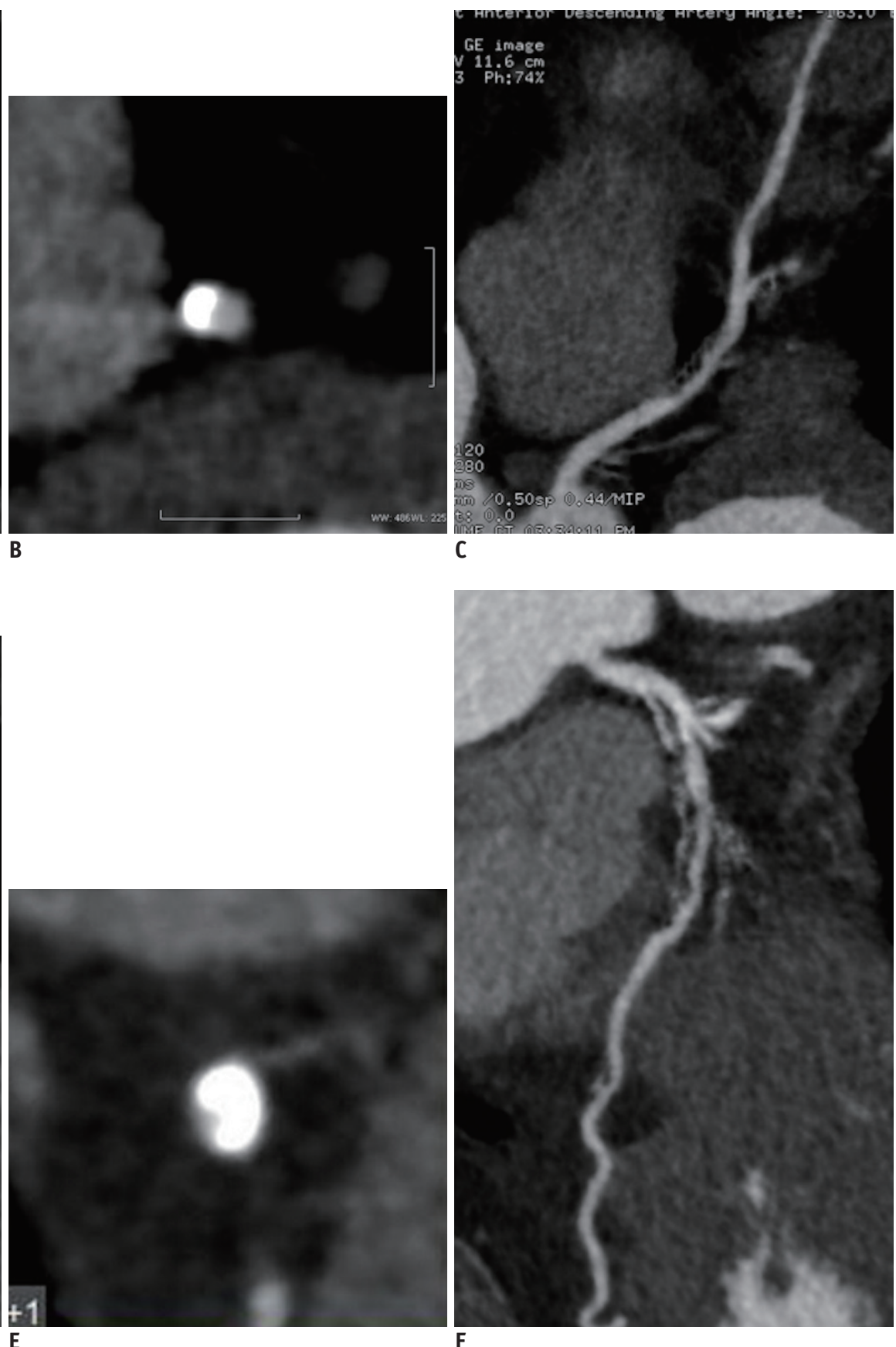

C

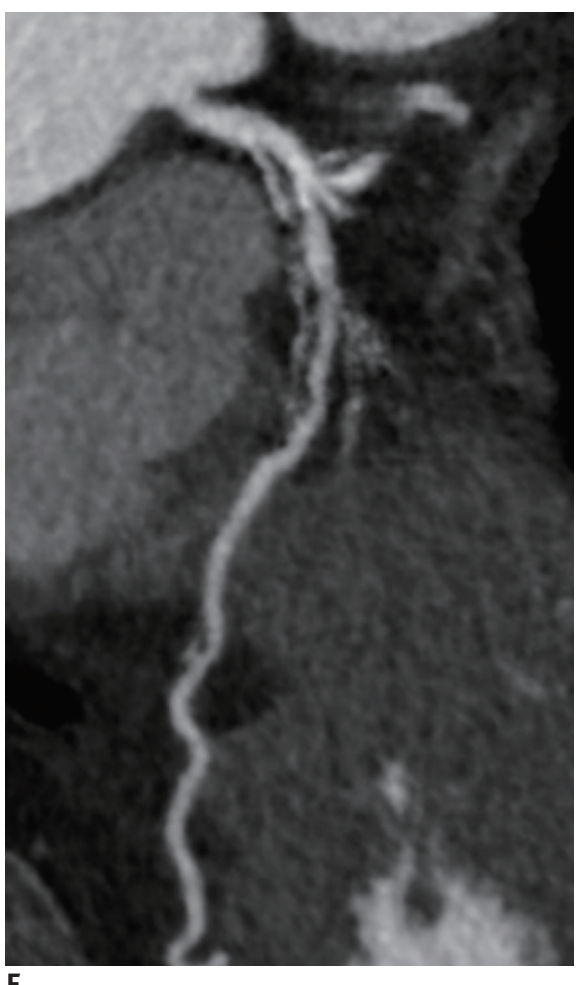

$\mathbf{F}$

\section{Fig. 4. Case showing stenosis regrading in conventional CCTA and subtraction.}

A, B. Moderate calcification is observed in left ascending artery on conventional images. C. After performing subtraction, calcification is eliminated. D-F. Degree of stenosis is assessed as mild in both subtraction and conventional CCTA. Corresponding subtraction process for severe plaque calcification at left anterior descending coronary artery from another patient. Moderate stenosis can be seen in subtraction CCTA, while severe stenosis is rated in conventional CCTA.

coronary segments with severe calcification of subtraction CCTA compared to conventional CCTA. Although increased misregistration artifacts were observed in segments with severe calcification, a relatively low frequency of misregistration was observed in this modified subtraction CCTA. For segments with severe calcification, the modified subtraction CCTA would change the stenosis grade and improve the diagnostic accuracy in comparison with conventional CCTA using ICA as the gold standard.

One of the challenges for conventional CCTA in stenotic evaluation is the presence of severe calcification or stents, which interfere with the visualization of the coronary 
Table 2. Diagnostic Accuracy of Conventional ССTA and Subtraction ССTA in Segments with Severe Calcification

\begin{tabular}{lccr}
\hline \multicolumn{1}{c}{ Measure } & Conventional CCTA & Subtraction CCTA & $P$ \\
\hline Sensitivity & $95 \%$ & $91 \%$ & 0.40 \\
Specificity & $70 \%$ & $87 \%$ & $<0.01$ \\
Positive predictive value & $61 \%$ & $79 \%$ & $<0.01$ \\
Negative predictive value & $96 \%$ & $94 \%$ & 0.70 \\
Accuracy & $79 \%$ & $89 \%$ & 0.05 \\
AUC $(95 \%$ CI $)$ & $0.85(0.74-0.96)$ & $0.94(0.86-1.00)$ & 0.03 \\
\hline
\end{tabular}

Segment-based analysis, $n=58$. AUC = area under curve, CCTA = coronary computed tomography angiography, $\mathrm{CI}=$ confidence interval

lumen and reduce the diagnostic accuracy. The specificity for diagnostic performance has been shown to decrease significantly in patients with severe coronary artery calcification in a multicenter study using a 64-detector CT (24). Subtraction CCTA using dedicated postprocessing software has been shown to improve diagnostic accuracy over conventional CCTA in patients with severe calcification $(9,10)$. Additionally, it has also been demonstrated that subtraction CCTA is useful in patients with coronary stents $(11,12,14,15)$.

In subtraction CCTA, one- and two-breathhold acquisition protocols have been used to obtain the mask image data. A one-breathhold approach requires a long breath-holding time, and even a modified one-breathhold scan protocol tested by Yoshioka et al. (13) required approximately 15 seconds of breath-holding time to provide diagnostic images. Many elderly patients with a high coronary artery calcium score and obstructive lung disease may not be able to hold their breath for such a long time. Moreover, misregistration artifacts have been shown to be more pronounced (approximately 50\% of all target segments) in previous two-breathhold approach studies due to different HR or breathhold variability between the noncontrast and the contrast data sets $(14,15)$. To further reduce the frequency of misregistration artifacts in subtraction CCTA, we propose a modified subtraction CCTA method with a twobreathhold technique.

In comparison with conventional CCTA, the modified subtraction CCTA protocol resulted in subtraction images with improved image quality. Misregistration artifacts were observed in $26 \%$ of target segments with severe calcification, which was significantly lower than the previous study (approximately 50\%) designed with the original two-breathhold method.

In our study, stenosis regrading mostly occurred in coronary segments with severe calcification, which implies that the subtraction technique would have more effective clinical significance for this type of calcification. In

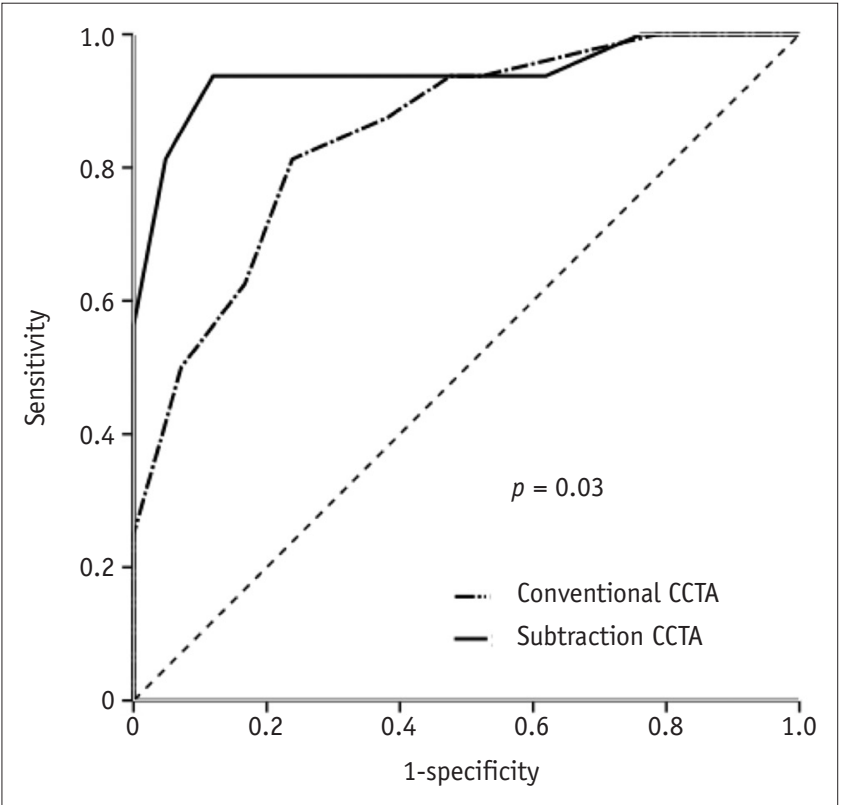

Fig. 5. Area under receiver operating characteristic curves of conventional CCTA and subtraction CCTA versus invasive coronary angiography: AUC for conventional CCTA is $0.85(95 \%$ CI, 0.74-0.96). AUC for subtraction CCTA is 0.94 ( $95 \%$ CI, 0.861.00). $A U C=$ area under curve, $C I=$ confidence interval

addition, the diagnostic accuracy of modified subtraction CCTA was improved by its increased specificity and positive predictive value for the assessment of coronary artery stenosis when compared to conventional CCTA. The AUC of the modified subtraction CCTA (0.94) was significantly higher than that of conventional CCTA (0.85) $(p=0.03)$. These results indicate that modified subtraction CCTA using a modified two-breathhold technique may have adequate accuracy in the diagnosis of significant stenosis in segments with severe calcification.

Some limitations of this study should be acknowledged. First, the small sample size with a small number of calcification segments limited the generalizability of our conclusions. Second, only patients with optimum HR $<65$ bpm were eligible for modified subtraction CCTA scanning. Third, misregistration artifacts specific to subtraction CCTA 


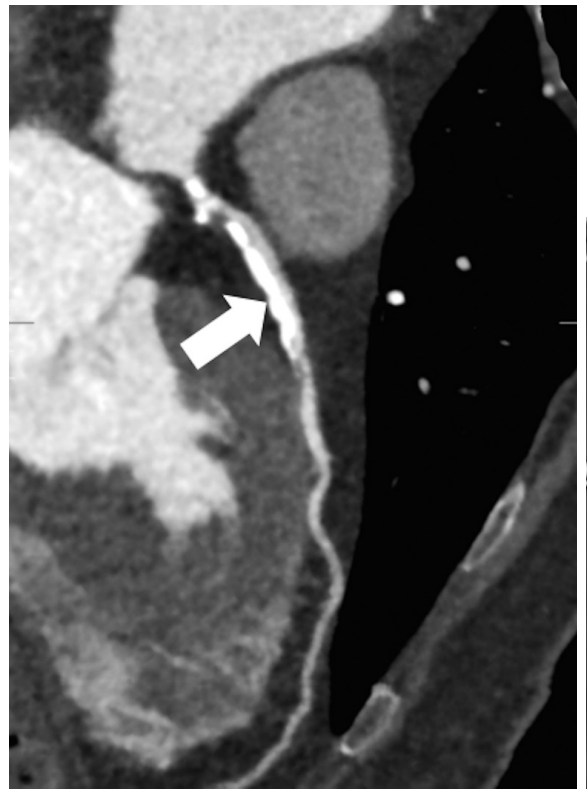

A

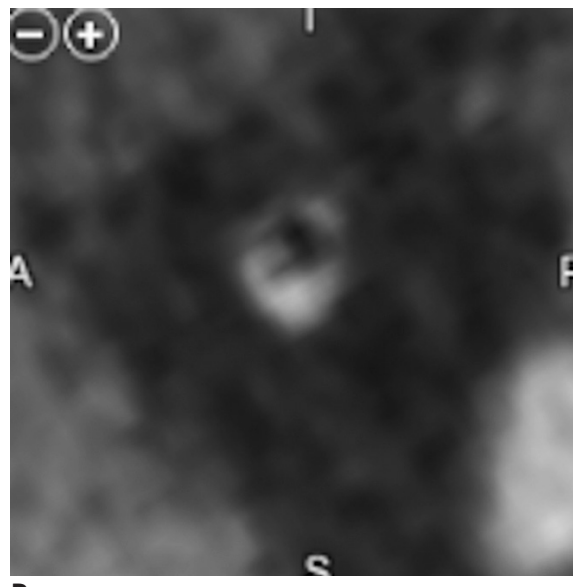

D

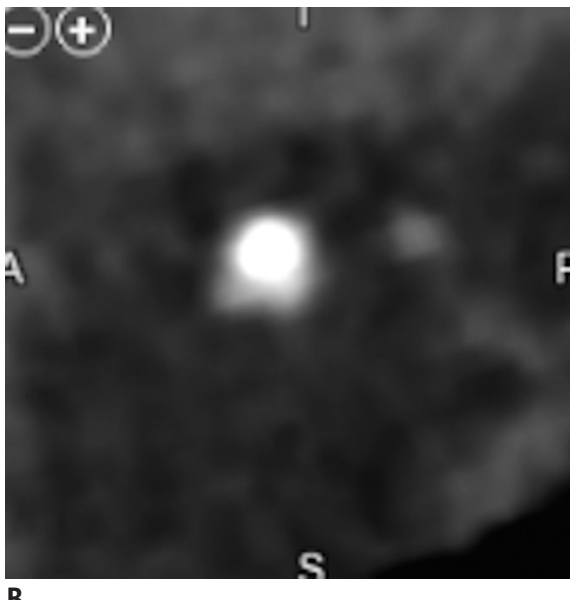

B

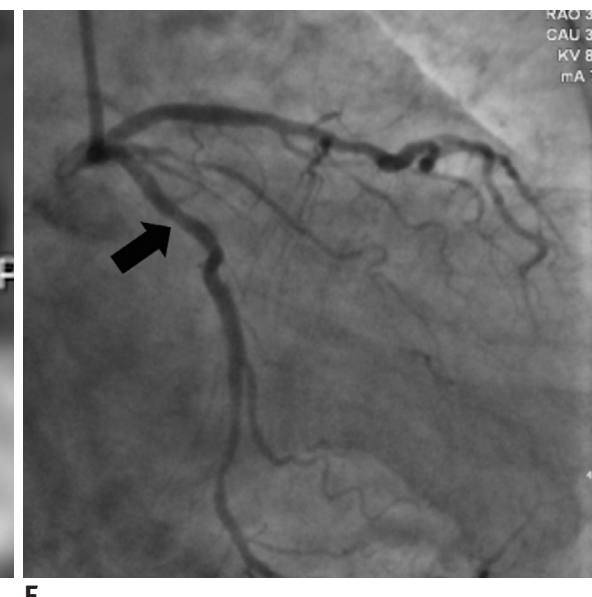

E

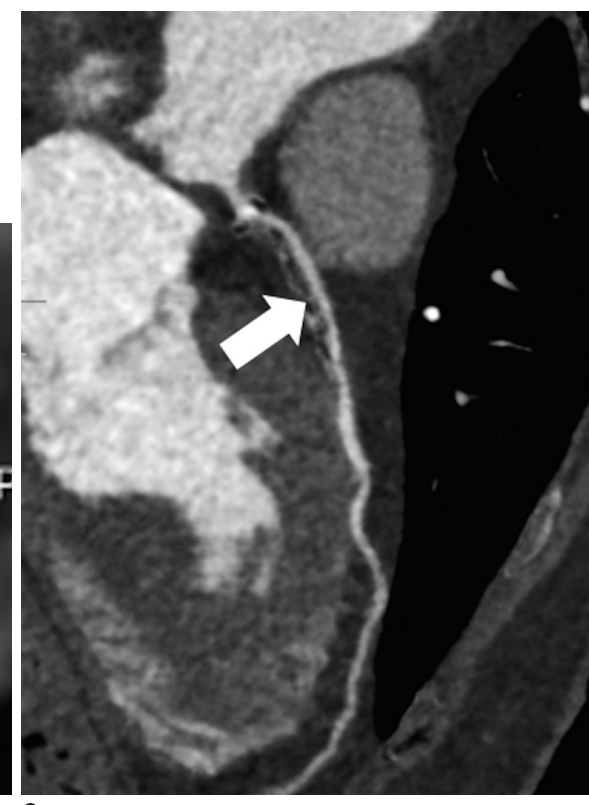

C

Fig. 6. 68-year-old man with suspected coronary artery disease.

From CPR image (A) and cross-sectional image (B) in conventional CCTA, severe calcification is observed in proximal portion of left anterior descending artery, which makes it difficult to assess lumen (arrow). In cross-sectional image (C) and CPR cross-sectional image (D) after subtraction, no significant stenosis is depicted (arrow). There is no significant stenosis determined by invasive coronary angiography (arrow) (E). $\mathrm{CPR}=$ curved planar reformation

are still generated in some calcified segments. The final limitation of modified subtraction CCTA is the relatively high radiation dose. In this study, only patients for whom prospective one-heartbeat scanning was possible were selected (25). The average total effective radiation dose in the present study was $5.5 \pm 1.7 \mathrm{mSv}$, which cannot be considered a low value.

To address the problems mentioned above, material density images using dual-energy CT have been reported to reduce or remove the depiction of coronary calcification (26). Theoretically, misregistration can be considered to be negligible due to the use of fast tube voltage switching. Therefore, if iodine and hydroxyapatite can be completely separated, this technique is an ideal method for the evaluation of severely calcified coronary artery segments. Additionally, some studies adopted novel advanced postprocessing algorithms, such as "sharpen" reconstruction (27) and the deblooming algorithm (28), to decrease the blooming artifacts from heavy calcification and consequently improve the diagnostic accuracy of CCTA in assessing coronary stenosis. Moreover, recent advances in computational fluid dynamics and individual imagebased modeling permit noninvasive calculation of fractional flow reserve derived from CCTA (FFR-CT). As demonstrated 
by Nørgaard et al. (29), FFR-CT provided higher diagnostic performance and discrimination of ischemia in patients with high calcification scores in comparison with CCTA assessment. In the future, noninvasive functional assessment based on CCTA will become an important tool for the diagnosis of coronary artery disease.

In summary, the results of this preliminary study have shown that the modified subtraction CCTA with a twobreathhold approach allows for stenosis regrading and improves the diagnostic accuracy in patients with segmentbased severe calcification and limited breath-holding capability.

\section{Conflicts of Interest}

The authors have no potential conflicts of interest to disclose.

\section{ORCID iDs}

Mengsu Zeng

https://orcid.org/0000-0001-6054-0824

Weifeng Guo

https://orcid.org/0000-0003-2377-3279

\section{REFERENCES}

1. Miller JM, Rochitte CE, Dewey M, Arbab-Zadeh A, Niinuma $\mathrm{H}$, Gottlieb I, et al. Diagnostic performance of coronary angiography by 64-row CT. N Engl J Med 2008;359:2324-2336

2. Dewey M, Vavere AL, Arbab-Zadeh A, Miller JM, Sara L, Cox $C$, et al. Patient characteristics as predictors of image quality and diagnostic accuracy of MDCT compared with conventional coronary angiography for detecting coronary artery stenoses: CORE-64 Multicenter International Trial. AJR Am J Roentgenol 2010;194:93-102

3. Meijboom WB, Meijs MF, Schuijf JD, Cramer MJ, Mollet NR, van Mieghem CA, et al. Diagnostic accuracy of 64-slice computed tomography coronary angiography: a prospective, multicenter, multivendor study. J Am Coll Cardiol 2008;52:2135-2144

4. Zhang S, Levin DC, Halpern EJ, Fischman D, Savage M, Walinsky P. Accuracy of MDCT in assessing the degree of stenosis caused by calcified coronary artery plaques. AJR Am J Roentgenol 2008;191:1676-1683

5. Dewey M. Coronary CT versus MR angiography: pro CT--the role of CT angiography. Radiology 2011;258:329-339

6. Palumbo AA, Maffei E, Martini C, Tarantini G, Di Tanna $\mathrm{GL}$, Berti $\mathrm{E}$, et al. Coronary calcium score as gatekeeper for 64-slice computed tomography coronary angiography in patients with chest pain: per-segment and per-patient analysis. Eur Radiol 2009;19:2127-2135

7. Ahn SJ, Kang DK, Sun JS, Yoon MH. Accuracy and predictive value of coronary computed tomography angiography for the detection of obstructive coronary heart disease in patients with an Agatston calcium score above 400. J Comput Assist Tomogr 2013;37:387-394

8. Yoshioka K, Tanaka R, Muranaka K. Subtraction coronary CT angiography for calcified lesions. Cardiol Clin 2012;30:93-102

9. Amanuma M, Kondo T, Sano T, Sekine T, Takayanagi $\mathrm{T}$, Matsutani $\mathrm{H}$, et al. Subtraction coronary computed tomography in patients with severe calcification. Int J Cardiovasc Imaging 2015;31:1635-1642

10. Yoshioka K, Tanaka R, Muranaka K, Sasaki T, Ueda T, Chiba $\mathrm{T}$, et al. Subtraction coronary CT angiography using secondgeneration 320-detector row CT. Int J Cardiovasc Imaging 2015;31 Suppl 1:51-58

11. Viladés Medel D, Leta R, Alomar Serralach X, Carreras Costa F, Pons-Lladó G. Reliability of a new method for coronary artery calcium or metal subtraction by 320-row cardiac CT. Eur Radiol 2016;26:3208-3214

12. Amanuma M, Kondo T, Sano T, Takayanagi T, Matsutani $H$, Sekine $T$, et al. Assessment of coronary in-stent restenosis: value of subtraction coronary computed tomography angiography. Int J Cardiovasc Imaging 2016;32:661-670

13. Yoshioka K, Tanaka R, Takagi H, Nagata K, Chiba T, Takeda K, et al. Diagnostic accuracy of a modified subtraction coronary CT angiography method with short breath-holding time: a feasibility study. Br J Radiol 2016;89:20160489

14. Fuchs A, Kühl JT, Chen MY, Helqvist S, Razeto M, Arakita K, et al. Feasibility of coronary calcium and stent image subtraction using 320-detector row CT angiography. J Cardiovasc Comput Tomogr 2015;9:393-398

15. Fuchs A, Kühl JT, Chen MY, Viladés Medel D, Alomar X, Shanbhag SM, et al. Subtraction CT angiography improves evaluation of significant coronary artery disease in patients with severe calcifications or stents-the C-Sub 320 multicenter trial. Eur Radiol 2018;28:4077-4085

16. Agatston AS, Janowitz WR, Hildner FJ, Zusmer NR, Viamonte M Jr, Detrano R. Quantification of coronary artery calcium using ultrafast computed tomography. J Am Coll Cardiol 1990;15:827-832

17. Funama $Y$, Utsunomiya D, Taguchi K, Oda S, Shimonobo T, Yamashita Y. Automatic exposure control at single- and dualheartbeat CTCA on a 320-MDCT volume scanner: effect of heart rate, exposure phase window setting, and reconstruction algorithm. Phys Med 2014;30:385-390

18. Hausleiter J, Meyer T, Hermann F, Hadamitzky M, Krebs M, Gerber TC, et al. Estimated radiation dose associated with cardiac CT angiography. JAMA 2009;301:500-507

19. Razeto M, Mohr B, Arakita K, Schuijf JD, Fuchs A, Kühl JT, et al. Accurate, fully-automated registration of coronary arteries for volumetric CT digital subtraction angiography. Proceedings of SPIE - The International Society for Optical Engineering;2014 March 21; San Diego, CA, USA

20. Vavere AL, Arbab-Zadeh A, Rochitte CE, Dewey M, Niinuma $\mathrm{H}$, Gottlieb I, et al. Coronary artery stenoses: accuracy of 
64-detector row CT angiography in segments with mild, moderate, or severe calcification--a subanalysis of the CORE64 trial. Radiology 2011;261:100-108

21. Cerci R, Vavere AL, Miller JM, Yoneyama K, Rochitte CE, Dewey $M$, et al. Patterns of coronary arterial lesion calcification by a novel, cross-sectional CT angiographic assessment. Int J Cardiovasc Imaging 2013;29:1619-1627

22. Raff GL, Abidov A, Achenbach S, Berman DS, Boxt LM, Budoff MJ, et al.; Society of Cardiovascular Computed Tomography. SCCT guidelines for the interpretation and reporting of coronary computed tomographic angiography. J Cardiovasc Comput Tomogr 2009;3:122-136

23. DeLong ER, DeLong DM, Clarke-Pearson DL. Comparing the areas under two or more correlated receiver operating characteristic curves: a nonparametric approach. Biometrics 1988;44:837-845

24. Abdulla J, Pedersen KS, Budoff M, Kofoed KF. Influence of coronary calcification on the diagnostic accuracy of 64-slice computed tomography coronary angiography: a systematic review and meta-analysis. Int J Cardiovasc Imaging 2012;28:943-953
25. Earls JP, Berman EL, Urban BA, Curry CA, Lane JL, Jennings RS, et al. Prospectively gated transverse coronary CT angiography versus retrospectively gated helical technique: improved image quality and reduced radiation dose. Radiology 2008;246:742-753

26. Machida H, Tanaka I, Fukui R, Shen Y, Ishikawa T, Tate E, et al. Current and novel imaging techniques in coronary CT. Radiographics 2015;35:991-1010

27. Sun Z, Ng CK, Xu L, Fan Z, Lei J. Coronary CT angiography in heavily calcified coronary arteries: improvement of coronary lumen visualization and coronary stenosis assessment with image postprocessing methods. Medicine (Baltimore) 2015;94:e2148

28. Li P, Xu L, Yang L, Wang R, Hsieh J, Sun Z, et al. Blooming artifact reduction in coronary artery calcification by a new de-blooming algorithm: initial study. Sci Rep 2018;8:6945

29. Nørgaard BL, Gaur S, Leipsic J, Ito H, Miyoshi T, Park SJ, et al. Influence of coronary calcification on the diagnostic performance of CT angiography derived FFR in coronary artery disease: a substudy of the NXT trial. JACC Cardiovasc Imaging 2015;8:1045-1055 